\title{
High contrast staining for serial block face scanning electron microscopy without uranyl acetate
}

\author{
Adolfo Odriozola ${ }^{1}$, Jaime Llodrá ${ }^{1,2}$, Julika Radecke ${ }^{1,5}$, Céline \\ Ruegsegger ${ }^{3}$, Stefan Tschanz ${ }^{1}$, Smita Saxena ${ }^{3,4}$, Stephan Rohr ${ }^{2}$, and \\ Benoît Zuber ${ }^{1, *}$ \\ ${ }^{1}$ Institute of Anatomy, University of Bern, Switzerland \\ ${ }^{2}$ Department of Physiology, University of Bern, Switzerland \\ ${ }^{3}$ Institute of Cell Biology, University of Bern, Switzerland \\ ${ }^{4}$ Department of Neurology, Inselspital, Bern University Hospital, \\ University of Bern, Switzerland \\ ${ }^{5}$ present address: Center for Neuroscience, University of Copenhagen, \\ Denmark \\ *corresponding author
}

October 22, 2017

\section{Abstract}

Serial block face scanning electron microscopy (SBFSEM) is an increasingly popular method for investigating the three-dimensional ultrastructure of large biological samples. Prior to imaging, samples are typically chemically fixed, stained with osmium and uranyl acetate, and subsequently embedded in resin. The purpose of staining is to provide image contrast and reduce specimen charging under the electron beam, which is detrimental to the quality of imaging. Obtaining, using, and disposing of uranyl acetate is getting increasingly cumbersome in many countries due to new regulations on the handling of radioactive substances. Therefore, we developed an alternative staining procedure that does not rely on the use of uranium or any other radioactive substance. This procedure provides excellent contrast and efficiently reduces specimen charging.

\section{Introduction}

By providing access to cell and tissue ultrastructure at nanometer resolution, transmission electron microscopy (TEM) has revolutionized cell biology in the mid-20th century. Yet classical TEM only provides 2-dimensional images, which hinders the assessment of the 3-dimensional cellular structure of the tissue under investigation. TEM analysis of serial sections has alleviated this problem but the throughput for producing, imaging, 
and processing serial sections of useful sample volume is very low. Recently two scanning electron microscopy (SEM) modalities have been introduced where a resin-embedded specimen block is placed in the microscope and its surface is repeatedly and automatically 'shaved' and imaged, producing serial images at a much higher throughput [1]. 'Shaving' is achieved by using either a focused ion beam (FIBSEM: focused ion beam SEM) or an ultramicrotome with a diamond knife, which is mounted inside the microscope specimen chamber (SBFSEM: serial block face SEM). SBFSEM can image larger volumes than FIBSEM although at somewhat lower resolution (20 to $30 \mathrm{~nm}$ vs. 5 to $10 \mathrm{~nm}$ ) [1, 2]. Note that these numbers refer to effective resolution and not to pixel size, which is often mistaken for resolution in the FIBSEM and SBFSEM fields. Resin-embedded specimens must be contrasted by heavy metal staining. While for TEM imaging specimen preparation typically involves a combination of in-block and on-section staining, FIBSEM and SBFSEM imaging depends exclusively on in block staining, which renders the procedure more difficult, since in FIBSEM and SBFSEM the block face, and not sections, is imaged. Aside its function in contrast establishment, heavy metal staining renders specimens electrically conductive. This counteracts specimen charging occurring under the scanning electron beam thereby leading to image distortion. Charging is a particular concern in SBFSEM, where it is inherently more prone to occur than in FIBSEM. Indeed it is thought that ion beam scanning neutralizes the charges induced by the electron beam scan. Furthermore charging has another deleterious consequence in SBFSEM as it weakens the resin and leads to fragmentation of the block surface during sectioning. A highly efficient heavy metal staining procedure is therefore of critical importance for samples to be imaged by SBFSEM.

Most staining protocols for SBFSEM consist of successive incubations with reduced osmium, a mordant for osmium, osmium tetroxide, uranyl acetate, and lead aspartate [3. 4, 5, 6. It is generally considered that osmium mainly stains membranes, whereas uranyl stains proteins and chromatin and lead enhances uranyl staining [7]. Working with uranyl acetate has become increasingly cumbersome in recent years due to the introduction of stricter regulations on radioactive material in several countries. In addition, some research institutions enforce more rigorous rules than the legal requirements in term of radioactive waste disposal. This results in time and cost intensive procedures. We therefore sought to develop an alternative staining protocol for SBFSEM that does not rely on the use of radioactive material. Lanthanides have been used as alternative on-section stain for TEM and have provided similar staining properties to uranyl acetate [8, 9]. On this basis we developed a protocol that uses lanthanides, namely samarium and gadolinium salts, instead of uranyl acetate during bloc staining. We tested the protocol on various samples. The results show that contrast is at least as good or improved, whereas charging is reduced.

\section{Results}

\subsection{Cerebellar cortex}

We sought to develop a bloc staining protocol free of radioactive uranyl acetate for SBFSEM (see Methods). Instead of uranyl acetate, we used gadolinium triacetate and samarium triacetate, which were previously shown to be efficient staining agents for ultrathin sections [8]. We tested the protocol on mouse cerebellar cortex. The contrast provided by this procedure was excellent (Figure 1). The two membranes composing the nuclear 
bioRxiv preprint doi: https://doi.org/10.1101/207472; this version posted October 22, 2017. The copyright holder for this preprint (which was not certified by peer review) is the author/funder, who has granted bioRxiv a license to display the preprint in perpetuity. It is made available under aCC-BY 4.0 International license.
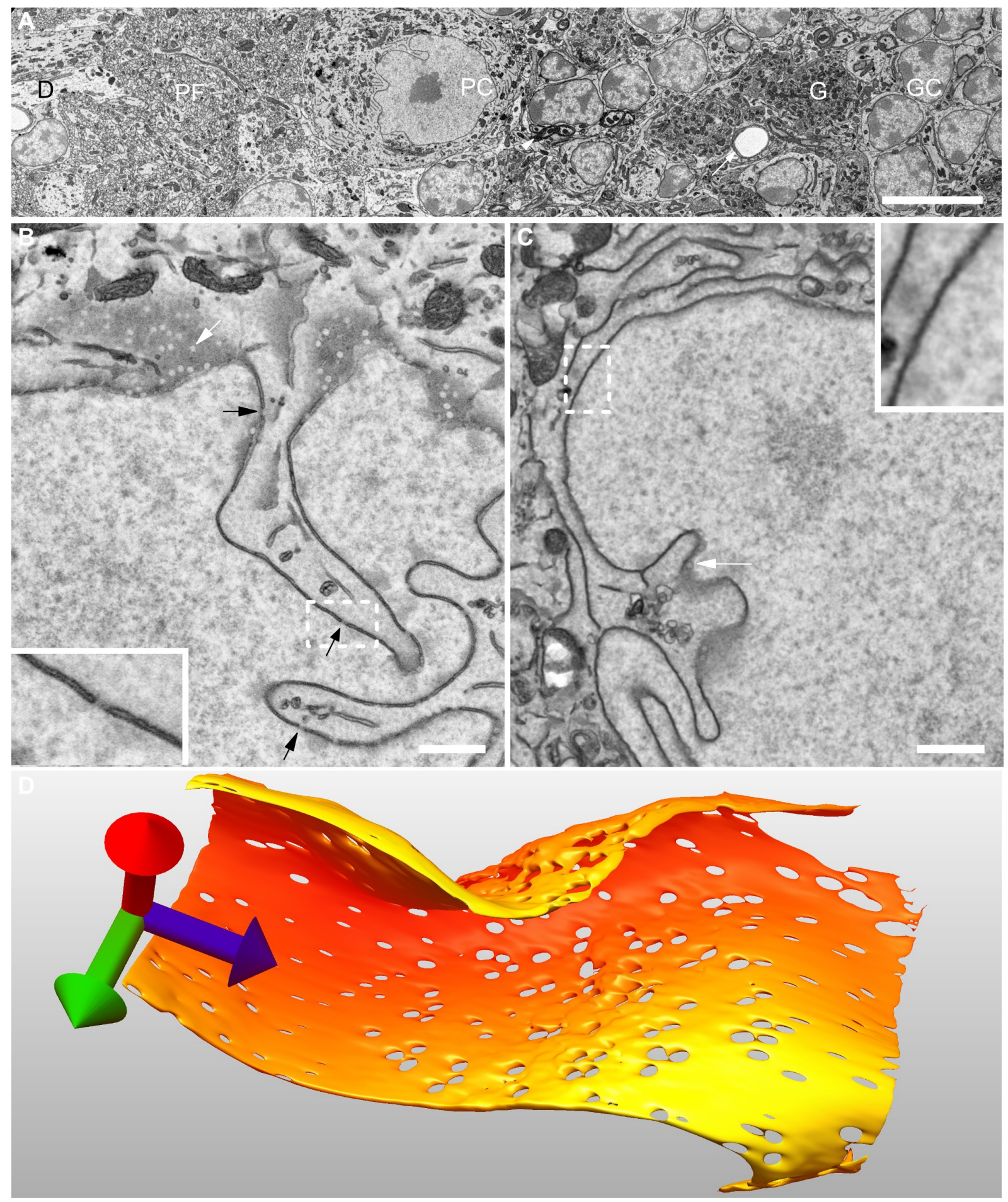

Figure 1: SBFSEM images of cerebellar cortex. A: Overview of cortex prepared with the lanthanide-based protocol shows excellent contrast. (D, Purkinje cell dendrites; PF, parallel fibers, PC, Purkinje cell body; G, cerebellar glomerulus; GC, granule cell body). B: High magnification view of a Purkinje cell nucleus prepared with the lanthanide protocol showing resolved nuclear membranes and nuclear pores in cross-section (black arrows and inset) and in grazing section (white arrows). 
Figure 1: continued. C: High magnification view of a Purkinje cell nucleus prepared with ROTO. The nuclear envelope appears as a single line (inset). Nuclear pores are only visible in grazing section (white arrow). D: Isosurface rendering of a portion of a Purkinje cell nuclear envelope prepared with the lanthanide protocol. The blue vector is perpendicular to the block face. Original pixel sizes: $40 \mathrm{~nm}(\mathrm{~A}), 3 \mathrm{~nm}(\mathrm{~B}), 6 \mathrm{~nm}(\mathrm{C})$, Bars: $10 \mu \mathrm{m}(\mathrm{A}), 1 \mu \mathrm{m}(\mathrm{B}, \mathrm{C})$
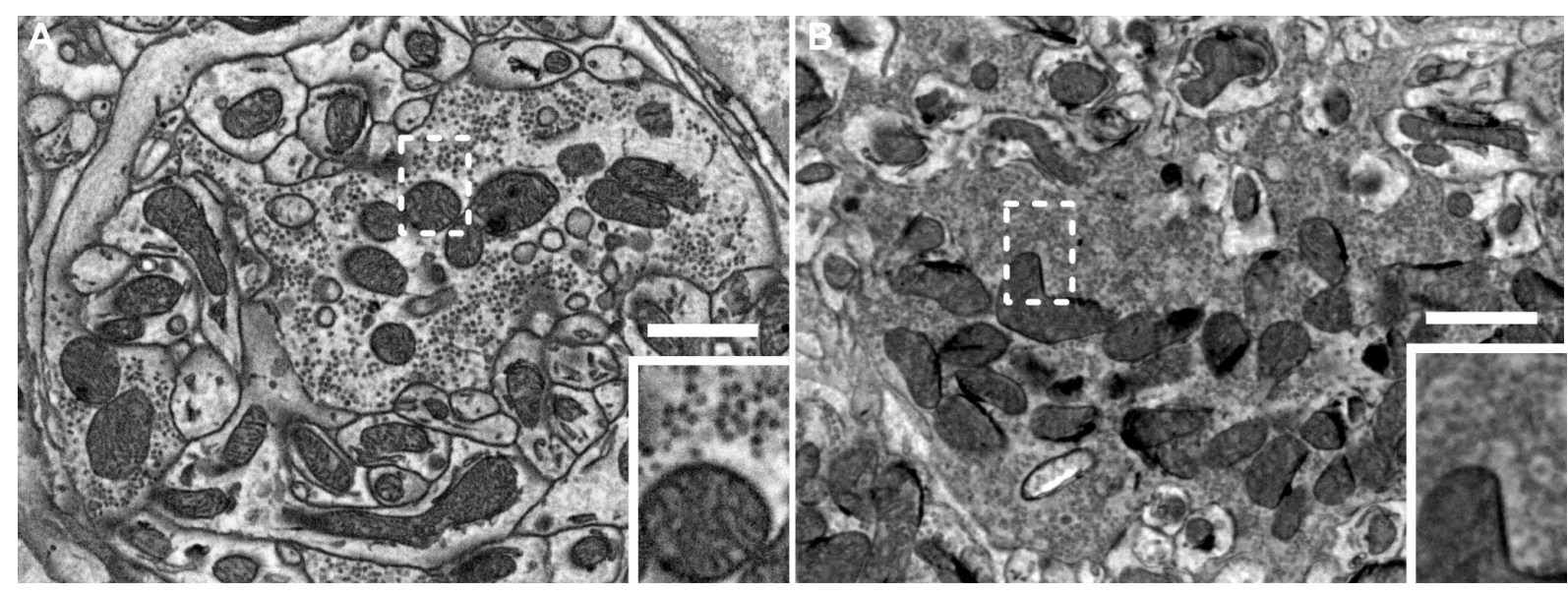

Figure 2: SBFSEM images of cerebellar cortex mossy fiber synapses. A: High magnification view of a mossy fiber synapse in a cerebellar glomerulus prepared with the lanthanide protocol. Synaptic vesicles, as well as mitochondrial cristae are clearly resolved (inset). B: High magnification image of a mossy fiber synapse prepared with the ROTO protocol. Synaptic vesicles are less well resolved and cristae are barely visible. Original pixel size: $5.5 \mathrm{~nm}$. Bars: $1 \mu \mathrm{m}$.

envelope, as well as the two membranes of endoplasmic reticulum cisternae were clearly resolved (Figure 1B and S1 Movie). On the other hand, nuclear membranes of specimens of samples processed with the ROTO protocol (using uranyl acetate as defined under Methods) appeared as a single line (Figure $1 \mathrm{C}$ ). Furthermore, with the ROTO protocol, nuclear pores were poorly visible in grazing section and practically unresolved in crosssection, whereas they were obvious with lanthanide staining, both in grazing section and in cross-section (arrows in Figure $1 \mathrm{~B}$ and $\mathrm{C}, \mathrm{S} 1 \mathrm{Movie}$ ). Contrast after lanthanide staining was such that the nuclear envelope and nuclear pores could be rendered in 3-dimension without the need of manual segmentation (Figure 1D). From such representation it is obvious that Purkinje cell nuclear pores are frequently found in small clusters of pores, consistent with a previous report [10]. Heavy electron radiation necessary to obtain high resolution SBFSEM images often generates chemical modification of the specimen leading to severe sectioning artefacts, which preclude acquisition of 3-dimensional stacks at high resolution [11]. These damages are especially pronounced in samples with low electrical conductivity. From the lanthanide-based protocol processed samples we could record high resolution stacks of hundreds of images with a slice thickness of $50 \mathrm{~nm}$ without any noticeable damages, indicating efficient staining resulting in good conductivity (S1 Movie). Moreover mitochondria cristae as well as synaptic vesicles were well resolved after lanthanide staining (Figure 2A, S1 Movie) whereas they were ill-defined after ROTO staining (Figure 2B). 

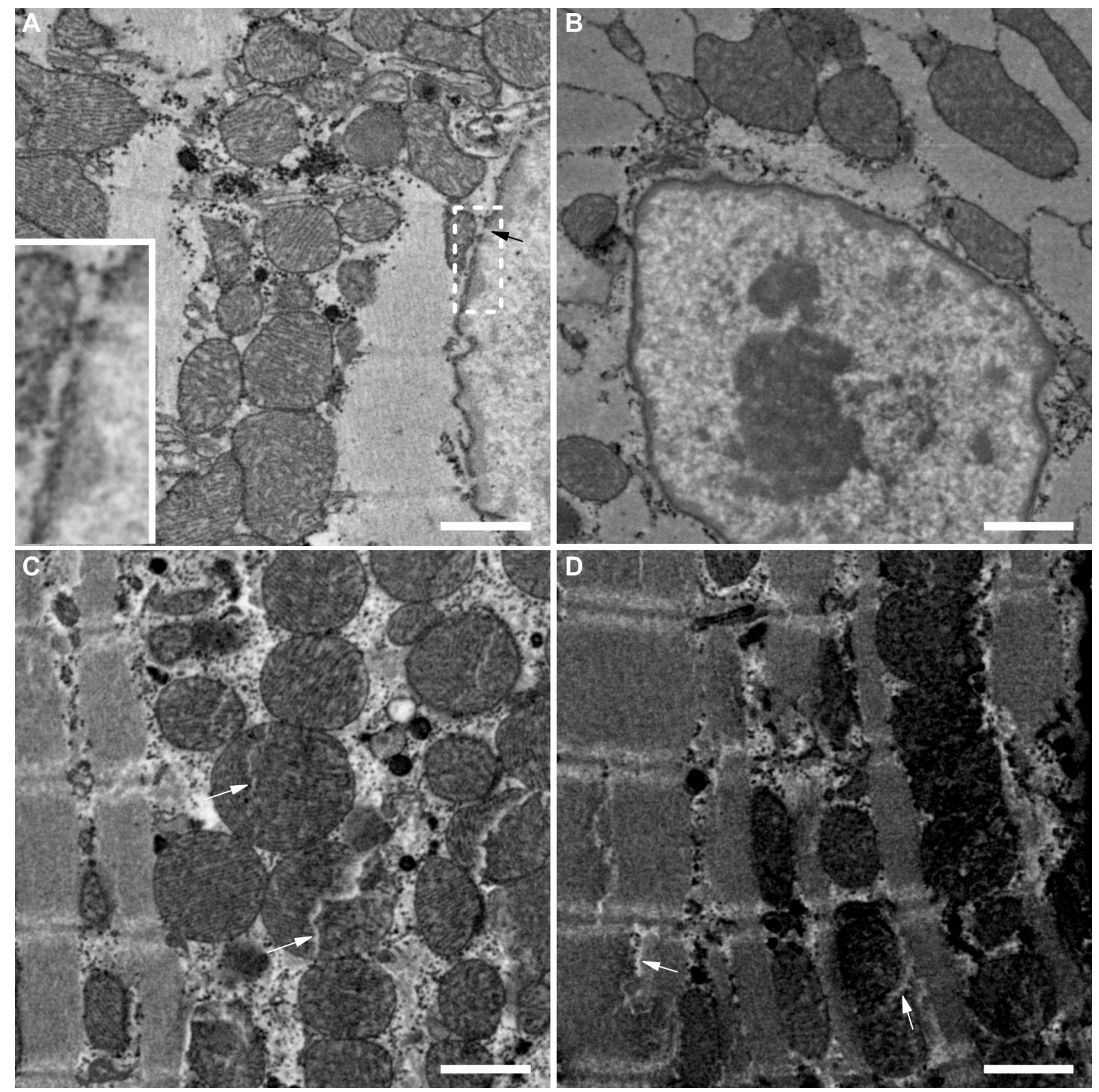

Figure 3: High magnification SBFSEM images of cardiomyocytes. A: Sample prepared with the lanthanide protocol. Cristae as well as nuclear pores (black arrow, inset) were well resolved. B: Sample prepared with the lanthanide protocol where the osmication steps were performed at room temperature. Nuclear pores are not resolved and cristae are hardly visible. C and D: Sample prepared with the lanthanide protocol with longer osmication steps at $50^{\circ} \mathrm{C}$. These two images show two fields of view, which are $6 \mu \mathrm{m}$ apart. The image in $\mathrm{C}$ shows good contrast whereas the area in $\mathrm{D}$ is too strongly stained. In the latter, mitochondria appear so dark that cristae are not visible. White arrows indicate cracks. Original pixel sizes: $6 \mathrm{~nm}(\mathrm{~A}-\mathrm{D})$. Bars: $1 \mu \mathrm{m}(\mathrm{A}-\mathrm{D})$.

\subsection{Myocardium}

We applied the lanthanide-based protocol to rat myocardium and we decided to assess on this tissue if the duration of the osmication steps performed at high temperature could influence the staining quality. First we applied the exact same osmication steps as for cerebellum $\left(\mathrm{OsO}_{4}\right.$ and potassium ferrocyanide incubation for 45 minutes at room temper- 
ature and then for 15 minutes at $50^{\circ} \mathrm{C}$, pyrogallol for 15 minutes at room temperature and for 5 minutes at $50^{\circ} \mathrm{C}$, and $\mathrm{OsO}_{4}$ for 22 minutes at room temperature and 8 minutes at $50^{\circ} \mathrm{C}$ ). As for cerebellum, the mitochondria cristae, nuclear membranes, and nuclear pores were resolved (Figure $3 \mathrm{~A}$ ). Furthermore, myofibrils and the sarcoplasmic reticulum were visible. These images, as those of cerebellar cortex, were acquired under high vacuum $(2 \mathrm{mPa})$. This implies that the samples were electrically conductive, in agreement with their strong staining. When all osmication steps were performed at room temperature $\left(\mathrm{OsO}_{4}\right.$ and potassium ferrocyanide incubation for 60 minutes, pyrogallol for 20 minutes, and $\mathrm{OsO}_{4}$ for 30 minutes, all at room temperature), myocardium samples were too lightly stained and not sufficiently conductive (Figure 3B). Hence they had to be imaged under low vacuum conditions (40 $\mathrm{Pa}$ ) to prevent charging. The individual membranes of the nuclear envelope could not be resolved at all. Nuclear pores were hardly visible and mitochondria cristae were far less well resolved than when osmication was performed at $50{ }^{\circ} \mathrm{C}$. Given the improved quality provided by osmication performed at $50^{\circ} \mathrm{C}$, we decided to increase the duration of the steps performed at $50^{\circ} \mathrm{C}$ with the aim of further improving contrast (incubation in $\mathrm{OsO}_{4}$ and potassium ferrocyanide for $60 \mathrm{~min}$ at $50^{\circ} \mathrm{C}$, incubation with pyrogallol for 20 minutes at $50^{\circ} \mathrm{C}$, and second incubation in $\mathrm{OsO}_{4}$ for $30 \mathrm{~min}$ at $50^{\circ} \mathrm{C}$ ). Some areas of the samples were well contrasted (Figure $3 \mathrm{C}$ ) whereas adjacent areas were too strongly stained (Figure $3 \mathrm{D}$ ). Consequently the contrast was low since the material was mostly dark. Note that in these, several cracks were visible indicating a too strong staining [12]. Even in the less strongly stained areas cracks were visible in mitochondria and their cristae were poorly resolved (Figure $3 \mathrm{C}$ ). Taken together these results demonstrate that the duration of the osmication steps can be optimized in order to obtain best contrasting and conducting performances.

\section{$3.3 \quad$ Lung}

Finally, we applied our protocol to rat lung and compared it to the ROTO procedure. The alveolar air compartment represents a large proportion of the sample and after specimen preparation it is filled with unstained resin. Consequently large areas of block surface are not conductive and imaging must be performed under low vacuum conditions (60 $\mathrm{Pa}$ ). The lanthanide protocol provided a better contrast. For example, vesicles and
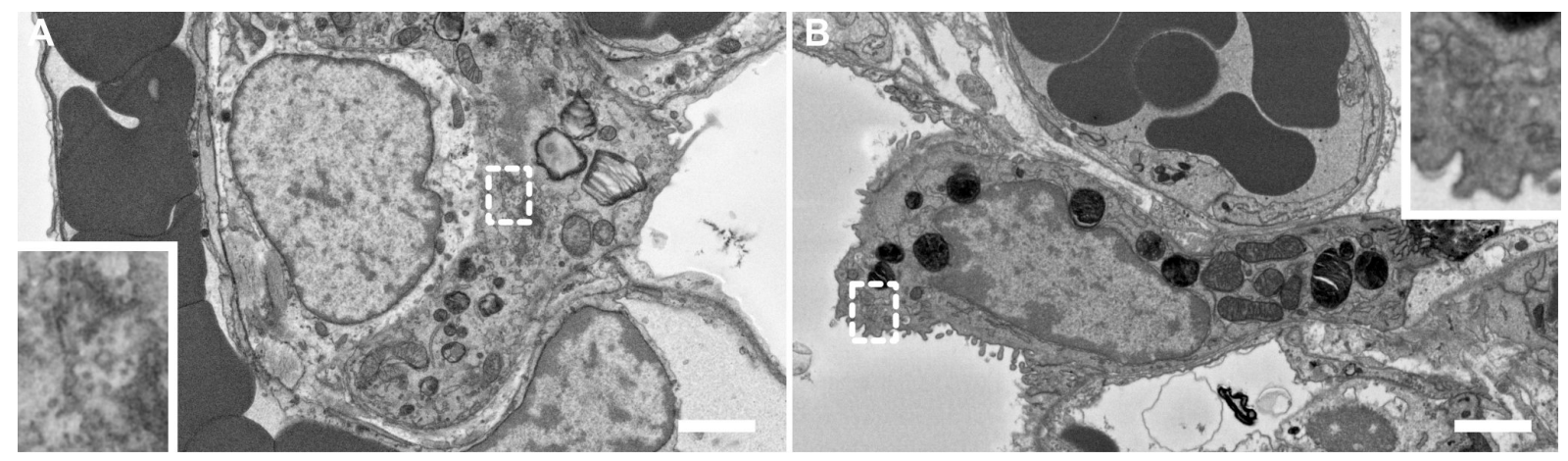

a

Figure 4: SBFSEM images of lung alveoli processed with the lanthanide protocol (A) or the ROTO protocol (B). On each image, a type II epithelial is visible. The contrast in these cells is slightly better after the lanthanide protocol (see insets). Original pixel sizes: $5.7 \mathrm{~nm}(\mathrm{~A}), 6 \mathrm{~nm}(\mathrm{~B})$. Bars: $2 \mu \mathrm{m}$ (A-D). 
other membranous compartment stood out better over cytoplasmic background and the contrast between hetero- and euchromatin was stronger (Figure 4). Lamellar bodies were saturated with stain after the ROTO procedure whereas they were more lightly stained and hence their inner structure was better resolved after the lanthanide protocol.

\section{Discussion}

SBFSEM has gained a lot of interest in the last couple of years. It allows fast acquisition of 3-dimensional ultrastructural information. Uranyl acetate is used in most procedures for sample staining prior to SBFSEM. Uranyl acetate is getting more and more difficult to obtain due to increasingly stricter regulations on radioactive substances. Hence we sought for a non-radioactive substitute that would efficiently stain biological samples for SBFSEM. Lanthanides salts have been successfully used to stain ultrathin sections for TEM [8, 9]. As they are not radioactive, we decided to perform en bloc staining with these salts. We applied them after osmication steps. Furthermore, we optimized osmication by performing some steps at $50{ }^{\circ} \mathrm{C}$. This resulted in excellent contrast allowing for example to clearly resolve the two nuclear membranes, nuclear pores, and mitochondrial cristae. In our hands, our new protocol provided significantly better staining than the uranylbased ROTO protocol. Most samples could be imaged under high vacuum, which is a prerequisite to reach the best possible resolution. Samples that contained a lot of nonstainable volume (e.g., air compartment in lung alveoli) needed to be imaged under low vacuum to prevent charging. Yet contrast in those samples was still very good. The protocol that we present here should be applicable to most biological samples. For each new type of specimen, we recommend to optimize the duration of the steps performed at $50^{\circ} \mathrm{C}$ as we demonstrated for myocardium. We anticipate that combining the new lanthanide-based staining protocol and sample embedding in conductive resins [6] will contribute to achieving optimal results.

\section{Methods}

All incubations in fixative were done at $4{ }^{\circ} \mathrm{C}$. All other steps were performed at room temperature except if indicated differently.

\subsection{Chemicals, cell culture, animals}

If not otherwise specified, chemicals were purchased from Sigma-Aldrich (Buchs, Switzerland). $\mathrm{OsO}_{4}$ and uranyl acetate were obtained from Electron Microscopy Sciences (Hatfield, PA, USA). Lung and heart samples were obtained from adult Wistar rats. Cerebellum samples were obtained from adult C57BL/6 mice. Milli-Q water was used to prepare all solutions and to perform all the rinses described in the steps below.

\section{$5.2 \quad$ Fixation}

A pneumothorax was applied to deeply anesthetized rats by diaphragm puncture and lungs were fixed by intratracheal application of ice-cold $2.5 \%$ glutaraldehyde in $0.15 \mathrm{M}$ HEPES [13, 14]. The lungs were then dissected out, immersed in fixative for 1 day, and chopped into small cubes measuring roughly $1 \mathrm{~mm}^{3}$. A thoracotomy was performed 
on deeply anesthetized adult Wistar rats. A piece of ventricle of approximately $1 \mathrm{~mm}^{3}$ was excised and immediately immersed in ice-cold $2.5 \%$ glutaraldehyde in $0.15 \mathrm{M}$ Nacacodylate solution, for 1 day. Deeply anesthetized mice were perfused with 0.1 M PBS and then with $2.5 \%$ glutaraldehyde $2 \%$ paraformaldehyde in $0.1 \mathrm{M}$ Na-cacodylate (all solution with $\mathrm{pH}$ 7.4). The cerebellum was isolated and immersed in the same solution overnight [15]. $180 \mu \mathrm{m}$ sections were produced with a vibratome.

\subsection{Staining}

The samples were stained following one of the two protocols described below.

\subsubsection{Staining protocol with uranyl acetate}

This protocol is known as reduced osmium, thiocarbohydrazide, osmium (ROTO) [16, 17]. Samples were rinsed $3 \times 5$ min in ice-cold $0.15 \mathrm{M}$ Na-cacodylate. They were then incubated in $0.15 \mathrm{M}$ Na-cacodylate solution containing $2 \% \mathrm{OsO}_{4}$ and $1.5 \%$ potassium ferrocyanide for $1 \mathrm{~h}$ at $4^{\circ} \mathrm{C}$. They were rinsed $3 \times 5 \mathrm{~min}$ with water, incubated for $20 \mathrm{~min}$ in $1 \%$ thiocarbohydrazide, rinsed again $3 \times 5$ min with water, and incubated in $2 \% \mathrm{OsO}_{4}$ for 30 min. They were rinsed $3 \times 5$ min with water, incubated overnight at $4{ }^{\circ} \mathrm{C}$ in $1 \%$ uranyl acetate, rinsed $3 \times 5$ min with water, incubated in 1\% Walton's lead aspartate [18] at $60{ }^{\circ} \mathrm{C}$ for $30 \mathrm{~min}$, and rinsed $3 \times 5$ min with water.

\subsubsection{Staining protocol with lanthanides}

Unless otherwise specified, the protocol based on lanthanides was the following. Samples were rinsed $3 \times 5 \mathrm{~min}$ in ice-cold $0.15 \mathrm{M}$ Na-cacodylate. They were then incubated in $0.15 \mathrm{M}$ Na-cacodylate solution containing $2 \% \mathrm{OsO}_{4}$ and $1.5 \%$ potassium ferrocyanide for $45 \mathrm{~min}$ at room temperature and for $15 \mathrm{~min}$ in a water bath at $50^{\circ} \mathrm{C}$. They were rinsed $3 \times 5 \mathrm{~min}$ in water. They were incubated with $0.64 \mathrm{M}$ pyrogallol for 15 minutes at room temperature and for 5 minutes in a water bath at $50{ }^{\circ} \mathrm{C}$, and subsequently rinsed $3 \times 5$ min with water. Osmium amplification by pyrogallol instead of TCH was shown to generate stronger staining in the core of extremely large samples (whole mouse brain) [19]. Note that all the samples that we investigated were considerably thinner. The samples were incubated in $2 \% \mathrm{OsO}_{4}$ for $22 \mathrm{~min}$ at room temperature and $8 \mathrm{~min}$ in a water bath at $50{ }^{\circ} \mathrm{C}$. After $3 \times 5$ min rinses in water, the samples were incubated overnight in a solution of 0.15 M gadolinium acetate (LFG Distribution, Lyon, France) and $0.15 \mathrm{M}$ samarium acetate (LFG Distribution) pH 7.0. They were then rinsed $3 \times 5$ min with water, incubated in $1 \%$ Walton's lead aspartate [18] at $60^{\circ} \mathrm{C}$ for $30 \mathrm{~min}$, and rinsed $3 \times 5 \mathrm{~min}$ with water.

\subsection{Dehydration, embedding, and mounting}

After staining, the samples were dehydrated in a graded ethanol series $(20 \%, 50 \%, 70 \%$, $90 \%, 100 \%, 100 \%)$ at $4{ }^{\circ} \mathrm{C}$, each step lasting $5 \mathrm{~min}$. They were then infiltrated with Durcupan resin mixed with ethanol at ratios of $1: 3(\mathrm{v} / \mathrm{v}), 1: 1$, and $3: 1$, each step lasting $2 \mathrm{~h}$. They were then infiltrated with pure Durcupan overnight. The samples were transferred to fresh Durcupan and the resin was polymerized for 3 days at $60^{\circ} \mathrm{C}$. Sample blocks were mounted on aluminum pins (Gatan, Pleasonton, CA, USA) with a conductive epoxy glue (CW2400, Circuitworks, Kennesaw, GA, USA). Care was taken to have osmicated material directly exposed at the block surface in contact with the glue in order to reduce 
specimen charging under the electron beam. Pyramids with a surface of approximately $500 \times 500 \mathrm{\mu m}^{2}$ were trimmed with a razor blade.

\subsection{Electron microscopy}

SBFSEM was performed with a Quanta FEG 250 microscope (FEI, Eindhoven, The Netherlands), equipped with a 3View2XP in situ ultramicrotome (Gatan). Section thickness was set between 50 and $100 \mathrm{~nm}$. Images were acquired with magnifications and image sizes resulting in pixel sizes at specimen level ranging from 3 to $40 \mathrm{~nm}$. Images were acquired in high vacuum mode $(1-4 \mathrm{mPa})$, except where indicated otherwise. Acceleration voltage ranged from 3 to $5 \mathrm{kV}$ and pixel dwell time was set between 0.5 and $16 \mu \mathrm{s}$.

\subsection{Data availability}

The original images used in Supplementary Video S1 will be available on the EMPIAR repository (http://www.ebi.ac.uk/pdbe/emdb/empiar/). All other datasets generated during and/or analysed during the current study are available from the corresponding author on reasonable request.

\section{Acknowledgment}

This project was supported by a Swiss National Science Foundation grant (\#163761 to BZ). Imaging was performed on equipment supported by the Microscopy Imaging Center (MIC), University of Bern, Switzerland.

\section{Author contributions}

A. O. designed the project, performed experiments, and analyzed data. J. L., J. R., C. R., and S. T. performed experiments. S. S and S. R contributed materials and analyzed data. B. Z designed, directed and supervised the project, analyzed data, and wrote the manuscript with feedback from all authors.

\section{References}

[1] G. Knott and C. Genoud, "Is EM dead?," Journal of Cell Science, vol. 126, no. Pt 20, pp. 4545-52, 2013.

[2] C. Villinger, H. Gregorius, C. Kranz, K. Hohn, C. Munzberg, G. von Wichert, B. Mizaikoff, G. Wanner, and P. Walther, "FIB/SEM tomography with TEM-like resolution for 3D imaging of high-pressure frozen cells.," Histochem Cell Biol, vol. 138, pp. 549-556, Oct 2012.

[3] T. Deerinck, E. Bushong, V. Lev-Ram, X. Shu, R. Tsien, and M. Ellisman, "Enhancing serial block-face scanning electron microscopy to enable high resolution 3-D nanohistology of cells and tissues," Microscopy and Microanalysis, vol. 16, no. SupplementS2, pp. 1138-1139, 2010. 
[4] A. T. Thomas J. Deerinck, Eric A. Bushong and M. H. Ellisman, "NCMIR methods for 3D EM: A new protocol for preparation of biological specimens for serial blockface scanning electron microscopy," 2010.

[5] T. Q. Thai, H. B. Nguyen, S. Saitoh, B. Wu, Y. Saitoh, S. Shimo, Y. H. Elewa, O. Ichii, Y. Kon, T. Takaki, K. Joh, and N. Ohno, "Rapid specimen preparation to improve the throughput of electron microscopic volume imaging for three-dimensional analyses of subcellular ultrastructures with serial block-face scanning electron microscopy," Med Mol Morphol, 2016.

[6] A. A. Wanner, C. Genoud, T. Masudi, L. Siksou, and R. W. Friedrich, "Dense EMbased reconstruction of the interglomerular projectome in the zebrafish olfactory bulb," Nat Neurosci, vol. 19, no. 6, pp. 816-25, 2016.

[7] M. J. Dykstra and L. E. Reuss, Biological electron microscopy: theory, techniques, and troubleshooting. New York: Kluwer Academic/Plenum Publishers, 2nd ed ed., 2003.

[8] M. Nakakoshi, H. Nishioka, and E. Katayama, "New versatile staining reagents for biological transmission electron microscopy that substitute for uranyl acetate," $J$ Electron Microsc (Tokyo), vol. 60, no. 6, pp. 401-7, 2011.

[9] N. Hosogi, H. Nishioka, and M. Nakakoshi, "Evaluation of lanthanide salts as alternative stains to uranyl acetate," Microscopy (Oxf), vol. 64, no. 6, pp. 429-35, 2015.

[10] L. M. Garcia-Segura, M. Lafarga, M. T. Berciano, P. Hernandez, and M. A. Andres, "Distribution of nuclear pores and chromatin organization in neurons and glial cells of the rat cerebellar cortex," J Comp Neurol, vol. 290, pp. 440-50, Dec 1989.

[11] B. Titze and C. Genoud, "Volume scanning electron microscopy for imaging biological ultrastructure," Biol Cell, vol. 108, pp. 307-323, Nov 2016.

[12] A. A. Wanner, M. A. Kirschmann, and C. Genoud, "Challenges of microtome-based serial block-face scanning electron microscopy in neuroscience," J Microsc, vol. 259, pp. 137-42, Aug 2015.

[13] S. A. Tschanz, A. N. Makanya, B. Haenni, and P. H. Burri, "Effects of neonatal high-dose short-term glucocorticoid treatment on the lung: a morphologic and morphometric study in the rat," Pediatr Res, vol. 53, no. 1, pp. 72-80, 2003.

[14] S. A. Tschanz, L. A. Salm, M. Roth-Kleiner, S. F. Barre, P. H. Burri, and J. C. Schittny, "Rat lungs show a biphasic formation of new alveoli during postnatal development," J Appl Physiol (1985), vol. 117, no. 1, pp. 89-95, 2014.

[15] C. Ruegsegger, D. M. Stucki, S. Steiner, N. Angliker, J. Radecke, E. Keller, B. Zuber, M. A. Rüegg, and S. Saxena, "Impaired mTORC1-dependent expression of Homer-3 influences SCA1 pathophysiology," Neuron, vol. 89, pp. 129-46, Jan 2016.

[16] M. C. Willingham and A. V. Rutherford, "The use of osmium-thiocarbohydrazideosmium (OTO) and ferrocyanide-reduced osmium methods to enhance membrane contrast and preservation in cultured cells," J Histochem Cytochem, vol. 32, no. 4, pp. $455-60,1984$. 
[17] J. C. Tapia, N. Kasthuri, K. J. Hayworth, R. Schalek, J. W. Lichtman, S. J. Smith, and J. Buchanan, "High-contrast en bloc staining of neuronal tissue for field emission scanning electron microscopy," Nat Protoc, vol. 7, pp. 193-206, Jan 2012.

[18] J. Walton, "Lead aspartate, an en bloc contrast stain particularly useful for ultrastructural enzymology," J Histochem Cytochem, vol. 27, pp. 1337-42, Oct 1979.

[19] S. Mikula and W. Denk, "High-resolution whole-brain staining for electron microscopic circuit reconstruction," Nat Methods, vol. 12, no. 6, pp. 541-6, 2015.

\section{Supplementary material}

Supplementary Video S1. 200-slices stack through lanthanide-stained cerebellum Purkinje layer. The sample presents no cutting damage. Figure 1D was rendered from a part of this stack. Field of view size: $10.414 \times 10.913 \mu \mathrm{m}^{2}$. 